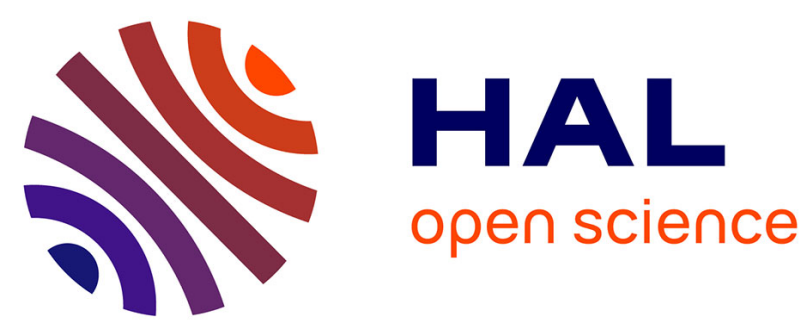

\title{
Phthalocyanine Photoregeneration for Low Power Consumption Chemiresistors
}

David Tomecek, Martin Hruska, Premysl Fitl, Jan Vlcek, Eva Maresova, Sarka Havlova, Lionel Patrone, Martin Vrnata

\section{To cite this version:}

David Tomecek, Martin Hruska, Premysl Fitl, Jan Vlcek, Eva Maresova, et al.. Phthalocyanine Photoregeneration for Low Power Consumption Chemiresistors. ACS Sensors, 2018, 3 (12), pp.25582565. 10.1021/acssensors.8b00922 . hal-02117021

\section{HAL Id: hal-02117021 \\ https://hal.science/hal-02117021}

Submitted on 1 May 2019

HAL is a multi-disciplinary open access archive for the deposit and dissemination of scientific research documents, whether they are published or not. The documents may come from teaching and research institutions in France or abroad, or from public or private research centers.
L'archive ouverte pluridisciplinaire HAL, est destinée au dépôt et à la diffusion de documents scientifiques de niveau recherche, publiés ou non, émanant des établissements d'enseignement et de recherche français ou étrangers, des laboratoires publics ou privés. 


\title{
Phthalocyanine Photoregeneration for Low Power Consumption Chemiresistors
}

\author{
David Tomecek,+ Martin Hruska,++ Premysl Fitl,++Jan Vlcek,+ Eva Maresova,+Sarka \\ Havlova,+ \\ Lionel Patrone, $\neq$ and Martin Vrnata*,+ \\ +University of Chemistry and Technology, Prague; Technicka 5, 16628 Prague 6 - Dejvice, Czech Republic \\ ‡Aix Marseille Univ., Universite 'de Toulon, CNRS, IM2NP UMR 7334, Yncre' a Me' diterrane 'e, ISEN \\ Toulon, Maison du Nume' rique \\ et de l'Innovation, Place G. Pompidou, 83000 Toulon, France \\ * Supporting Information
}

ABSTRACT: It is well-known that the applicability of phthalocyanine chemiresistors suffers from long recovery time after $\mathrm{NO}_{2}$ exposure. This circumstance enforces the necessity to operate the sensors at elevated temperatures $\left(150-200^{\circ} \mathrm{C}\right)$, which shortens the sensor lifetime and increases its power consumption (regardless, a typical measurement period is longer than $15 \mathrm{~min}$ ). In this paper, we propose a new method for fast and effective recovery by UV-vis illumination at a low temperature $\left(55^{\circ} \mathrm{C}\right)$. The method is based on short illumination following short $\mathrm{NO}_{2}$ exposure. To support and optimize the method, we investigated the effects of light in the wavelength and intensity ranges of $375-850 \mathrm{~nm}$ and $0.2-0.8 \mathrm{~mW} / \mathrm{mm} 2$, respectively, on the rate of $\mathrm{NO}_{2}$ desorption from the phthalocyanine sensitive

layer during the recovery period. This investigation was carried out for a set of phthalocyanine materials (ZnPc, $\mathrm{CuPc}, \mathrm{H}_{2} \mathrm{Pc}$,

$\mathrm{PbPc}$, and $\mathrm{FePc})$ operating at slightly elevated temperatures $\left(55-100^{\circ} \mathrm{C}\right)$ and was further supported by the analysis of UV-vis

and FTIR spectral changes. We found out that the light with the wavelength shorter than $550 \mathrm{~nm}$ significantly accelerates the

$\mathrm{NO}_{2}$ desorption from $\mathrm{ZnPc}, \mathrm{CuPc}$, and $\mathrm{FePc}$, and allows bringing the measurement period under 2 min and decreasing the

sensor power consumption by 75\%. Possible mechanisms of the light-stimulated desorption are discussed.

KEYWORDS: phthalocyanine chemiresistors, nitrogen dioxide detection, photoregeneration, lightstimulated $\mathrm{NO}_{2}$ desorption,

photoinduced spectral changes, low power consumption

$\mathrm{C}_{0}$ onventionally, $\mathrm{NO}_{2}$ is monitored by sensitive layers of

chemiresistors based on metal oxides.1 The best metal oxide-based chemiresistors are small, durable, and very sensitive electronic elements with detection limits as low as several parts per billion (ppb). 2 However, the metal oxide sensors operate at elevated temperatures $\left(>200{ }^{\circ} \mathrm{C}\right)$ and suffer from low selectivity to $\mathrm{NO}_{2.1}$ To overcome these obstacles, many researchers have focused on the investigation of organicbased sensors or organic-inorganic hybrids.

Organic materials offer a higher degree of compositional and structural variability that enables the sensor to be tailored to one selective application. Various materials such as metalorganic frameworks, 3,4 graphene composites, 5 or chemically 
derived carbon nanotubes, 6 polypyrrole, 7 porphyrin,8 polytoluidine, 9 and indigo-carmine 10 have already been investigated. Phthalocyanines (Pcs), which can operate at ambient temperatures, are also highly promising materials.11-14 Phthalocyanine $\left(\mathrm{H}_{2} \mathrm{Pc}\right)$ is an organic macrocyclic molecule with a summary formula $\left(\mathrm{C}_{8} \mathrm{H}_{4} \mathrm{~N}_{2}\right)_{4} \mathrm{H}_{2}$. It consists of four isoindole heterocycles connected by nitrogen bridges into one macrocycle with four nitrogen atoms in the center. The cavity in the center can be occupied by dozens (more than 70) of cation types forming phthalocyanine derivatives, metallophthalocyanines (MPc).15 They are typically intensively colored compounds absorbing UV-vis light by four absorption bands ( $Q$, B, N, and L). The light absorption promotes electrons across a band gap to a conduction band, generating new mobile charge carriers in phthalocyanines. 15 Phthalocyanine molecules contain an extended network of conjugated double bonds that interact with $\mathrm{NO}_{2}$. For such interaction, two basic mechanisms have been proposed: (a) the partial oxidation of the macrocycle connected with the introduction of free holes, 16,17 and (b) the formation of delocalized common molecular orbitals that significantly modify the mechanism of charge transport.18 In either case, the interaction results in a change of resistance (and impedance) that can be correlated with analyte concentration. ${ }_{19}$ Regarding the gas-sensing characteristics of Pc films, their morphology also plays a significant role.20 The most Received: August 28, 2018

Accepted: November 15, 2018

Published: November 15, 2018

Cite This: ACS Sens. 2018, 3, 2558-2565 pubs.acs.org/acssensors (C) 2018 American Chemical Society 2558 DOI: 10.1021/acssensors.8b00922 ACS Sens. 2018, 3, 2558-2565

Downloaded via AIX-MARSEILLE UNIV on January 2, 2019 at 22:27:20 (UTC).

See https://pubs.acs.org/sharingguidelines for options on how to legitimately share published articles. familiar phthalocyanine polymorphs are $\alpha, \beta, \chi$ and $\tau$. The first two, the $\alpha$ and $\beta$ forms, are common in phthalocyanine thin films.15,21 In particular, $\mathrm{NO}_{2}$ detection by various $\mathrm{Pc}$ morphologies was reported in ref 22.

However, the strong interaction of Pcs with $\mathrm{NO}_{2}$ (bonding energy $\approx 0.4 \mathrm{eV}$ ) results in long recovery times, which imposes serious limits on the real applicability of the sensors.17,23 While the effects of short-time and low-concentration exposures can usually be reversed by temperature elevation $\left(150-200^{\circ} \mathrm{C}\right)$ in a stream of pure air,24 longer exposures to higher concentrations even lead to irreversible changes in sensor properties. These changes were ascribed to the gradual transformation of the original $\mathrm{Pc}+\mathrm{NO}_{2}$ - complex to other species with an even stronger bonding.17 The stronger bonding "freezes" the thermal recovery mechanism as the hypothetical recovery temperature rises above the phthalocyanine degradation temperature. To mitigate the risk of sensor damage, the exposure times and doses were shortened and decreased by many researchers.24-26 Other papers suggested nanostructuring of Pcs or a kind of $\mathrm{NO}_{2}$ pre-exposure or preannealing to facilitate the $\mathrm{NO}_{2}$ release.27-29 Another approach that was demonstrated on $\mathrm{Ti}(\mathrm{Pc})_{2}$ and $\mathrm{Lu}(\mathrm{Pc})_{2}$ consisted of the application of UV light to accelerate the recovery.30,31 To avoid the long recovery times or sensor degradation at 
elevated temperatures, we propose to use and to tune the last strategy: the application of intensive light in UV and visible regions to stimulate the $\mathrm{NO}_{2}$ desorption (see Video $\mathrm{S} 1$ ) at an ambient or only slightly elevated temperature $\left(55-100{ }^{\circ} \mathrm{C}\right)$. So far, the illumination has been carried out either by using a UV mercury lamp with a broad spectrum with multiple wavelengths (applied at once) or only one narrow-band LED in the UV region at a single illumination intensity. 30,31 Therefore, in this work, we investigate the photoregenerating effects of light with various wavelengths in the range of 375$850 \mathrm{~nm}$ on various phthalocyanine derivatives ( $\mathrm{ZnPc}, \mathrm{CuPc}$, $\mathrm{H}_{2} \mathrm{Pc}, \mathrm{PbPc}$, and $\mathrm{FePc}$ ) at various intensities. We show that certain wavelengths in the UV-vis region (depending on the phthalocyanine derivative) are quite effective in the stimulation of $\mathrm{NO}_{2}$ desorption, thus shortening the recovery time by up to $90 \%$.

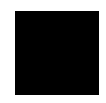

\section{EXPERIMENTAL SECTION}

Preparation of the Phthalocyanine Sensitive Layers.

Phthalocyanine chemiresistors were prepared by organic molecular evaporation $(\mathrm{OME})$ in high vacuum conditions $(\approx 1 \times 10-4 \mathrm{~Pa})$ on planar $2.5 \times 2 \mathrm{~mm} 2$ alumina substrates equipped with two interdigital Pt electrodes ( $25 \mu \mathrm{m}$ in width, $35 \mu \mathrm{m}$ far from each other). 32 Phthalocyanine powders were bought from Sigma-Aldrich and used for the chemiresistors without further purification. The evaporation of phthalocyanines (ZnPc, CuPc, $\mathrm{H}_{2} \mathrm{Pc}, \mathrm{PbPc}$, and $\mathrm{FePc}$ ) was carried out at various temperatures and growth rates summarized in Table S1. Phthalocyanine layers ( $250 \mathrm{~nm}$ thick) grew at ambient temperature on substrates that were situated $22.5 \mathrm{~cm}$ from the evaporation crucible. Thicknesses of the chemiresistor layers were measured with a profilometer (Kla Tencor P-6) and microscope (Tescan FE-SEM MIRA). The layers were further characterized by optical and IR spectroscopies (HR2000+ and Nicolet iS5).

Parameters of the Photoregeneration and Sensor Operation

Details. Chemiresistor sensing characteristics were measured on pretreated sensor samples after thermal stabilization at $100{ }^{\circ} \mathrm{C}$ and double photoinitiation by light with wavelength $(\lambda)$ of $441 \mathrm{~nm}$ and intensity (G) of $0.8 \mathrm{~mW} / \mathrm{mm} 2$ for $30 \mathrm{~s}$. The pretreatment was used in order to minimize their baseline (i.e., $\mathrm{R}_{0}=$ resistance in the synthetic air as a reference atmosphere) shift during the measurement. Sensor resistance was measured with an Agilent $34410 \mathrm{~A}(\mathrm{R}<1.5 \mathrm{G} \Omega$ ) or

Keithley 6517B $(\mathrm{R}>1.5 \mathrm{G} \Omega$ ) apparatus in a constant gas flow of 25 $\mathrm{mL} / \mathrm{min}$. Sensing characteristics were investigated for each combination of phthalocyanine and illumination wavelength (i.e., $5 \times 13$ matrix) by the application of testing sequences described in the following protocol:

1. Stabilization $=$ resistance stabilization in the reference atmosphere (the criterion for the stability was $\Delta R / R \leq 1 \%$ / min), 0-10 min.

2. Sensing = exposure to $0.2-1.6 \mathrm{ppm}$ of $\mathrm{NO}_{2}$ in the reference atmosphere, $30 \mathrm{~s}$.

3. Rinsing = exposure to the reference atmosphere to rinse unbonded $\mathrm{NO}_{2}, 10-30 \mathrm{~s}$.

4. Photoregeneration = chemiresistor illumination by light, $30 \mathrm{~s}$. 5. Evaluation $=$ did the resistance returned to its original value (step 1)? Yes = go to step 6; No = go back to step 4, $30 \mathrm{~s}$. 6 . Repetition = going back to step 1 to investigate repeatability $(\min 3 x)$. 
The sequence protocol is also shown in Figure 1. The individual sequence steps 1-6 are marked in the figure. The chemiresistor characteristics were calculated from the testing sequences and expressed as two quantities: normalized response magnitude NRM $=\left(\mathrm{Rb}_{b}-\mathrm{R}_{\mathrm{s}}\right) / \mathrm{Rb}$ and regeneration degree $\mathrm{RD}=\left(\mathrm{R}_{\mathrm{r} 1}-\mathrm{R}_{\mathrm{s}}\right) /\left(\mathrm{R}_{\mathrm{b}}-\mathrm{R}_{\mathrm{s}}\right)$. The NRM is a relative change of the sensor resistance during the exposure to $\mathrm{NO}_{2}$ and represents sensor sensitivity. The $\mathrm{RD}$ is a relative return of the resistance to its baseline representing the degree of sensor recovery after the $\mathrm{NO}_{2}$ exposure.

The photoregeneration of chemiresistors (step 4) was carried out by power LEDs (375-850 nm) refocused by two-lens optics to reach maximal spatial homogeneity of light intensity on the sensor (better than $\pm 5 \%$ for the LEDs except $500 \mathrm{~nm}$; for $500 \mathrm{~nm}$, better than $\pm 8 \%$ ). The illumination setup is depicted in Figure 2. All diodes were spectrometrically and radiometrically characterized by using a spectrophotometer (HR2000+), a calibrated UV-vis reference source (DH-2000), and an optical fiber with a cosine corrector. Diode operating conditions were adjusted to obtain four different average light intensities $(G)$ on the chemiresistors $(G=0.2,0.4,0.6$, and 0.8 $\mathrm{mW} / \mathrm{mm} 2$ ). Further details about the illumination are available in Table S2 and Figure S1 in the Supporting Information. Chemiresistor temperature was monitored and controlled by using an integrated Pt heater at the back side of the ceramic substrate. The chemiresistors were tempered at $55,70,85$, or $100{ }^{\circ} \mathrm{C}$ excluding step 4 in the testing sequence. During step 4 , the temperature mildly raised as a result of light absorption $\left(\mathrm{t}_{\mathrm{i}}-\mathrm{t}_{\mathrm{o}}=\max +8^{\circ} \mathrm{C}\right.$, see Figures 1 and 6). During that step, we kept the constant heating power equal to the

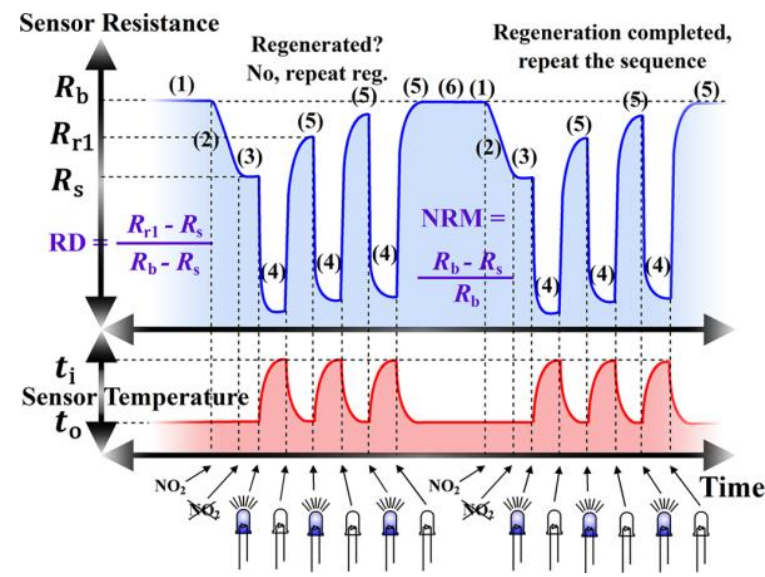

Figure 1. Testing sequence for the investigation of phthalocyanine photoregeneration. The individual sequence steps are marked with numbers: (1) stabilization, (2) sensing, (3) rinsing, (4) photoregeneration, (5) evaluation, and (6) repetition.

value from the previous step. This approach allowed to us effectively avoid the artifacts in resistance signals that originate in the PID controller's reaction to the change of the temperature. In the end, we repeated spectroscopic characterizations of the chemiresistive layers to compare the spectra before and after illuminations ( $\mathrm{G}=0.8 \mathrm{~mW}$ / $\mathrm{mm}_{2}$ for $2 \mathrm{~h}$ and $\mathrm{G}=7.3 \mathrm{~mW} / \mathrm{mm}_{2}$ for $1 \mathrm{~h}, \lambda=441 \mathrm{~nm}$ ).

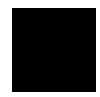

RESULTS AND DISCUSSION

The chemiresistor characteristics were calculated as two 
quantities, NRM and RD (defined above), obtained from the testing sequences measured for each combination of phthalocyanine (MPc) and wavelength $(\lambda)$ at various temperatures $(\mathrm{T})$, illumination intensities (G), and concentrations (c). Photoregeneration of $\mathrm{ZnPc}$ Chemiresistors at Various Temperatures, Illumination Intensities, and $\mathrm{NO}_{2}$ Concentrations. Photoregeneration at Various Temperatures. ZnPc chemiresistors were photoregenerated with certain effectivity by all the wavelengths ( $R D \gg 0 \%$ ). However, the regeneration degree (RD) significantly depended on the operating temperature and sensor baseline for the measurement. For this reason, the baseline value was always indicated and kept uniform for all measurements at the selected operating temperatures. Under these conditions, it was revealed that the regeneration degree (i.e., the effectivity of the photoregeneration process) increased with descending wavelength. For the temperature of $55^{\circ} \mathrm{C}$ and baseline of (320 \pm 9$)$

$\mathrm{M} \Omega$, the wavelengths longer than $550 \mathrm{~nm}$ had an approximately constant RD in the range from $10 \%$ to $17 \%$. (The intervals of the baseline values were constructed as a mean value of $\mathrm{Rb}_{\mathrm{b}} \pm$ corrected sample standard deviation of $\mathrm{Rb}$. The same methodology was also applied for the NRM intervals construction.) We labeled this region as the low effectivity photoregeneration (LEP) region due to the small effects of the illumination on the resistance recovery to its original values. From $550 \mathrm{~nm}$ going down, the RD was increasing. It went from $35 \%$ for $518 \mathrm{~nm}$ to $96 \%$ for $375 \mathrm{~nm}$ (see the black curve in Figure 3a). This region is further referred to as the high effectivity photoregeneration (HEP) region. This character of the $\mathrm{RD}=\mathrm{RD}(\lambda)$ dependence and the separation of RD values in two regions was preserved even if the operation temperature was altered. The values just slightly dropped. For the temperatures of 70,85 and $100{ }^{\circ} \mathrm{C}$ with the corresponding baselines $(301 \pm 10) \mathrm{M} \Omega$, $(302 \pm 13) \mathrm{M} \Omega$, and $(252 \pm 8)$ $\mathrm{M} \Omega$, the RD stayed in the intervals of (9-16\%), (7-12\%), and $(<12 \%)$, respectively, in the LEP region. In the HEP region, the RD decreased more significantly with the increasing temperature, providing the intervals of (33-81\%), (24-59\%), and (20-50\%), respectively (Figure 3a). At the same time, the $\mathrm{NRM}$ increased with temperature from $0.25 \pm 0.02$ for $55^{\circ} \mathrm{C}$ to $0.55 \pm 0.02$ for $100{ }^{\circ} \mathrm{C}$, indicating repeatable increase of sensitivity. On the contrary, for the series of measurements at one temperature, the NRM was preserved approximately constant for all sequences with no trend. This should mean that the state of the films was not significantly changing between the consecutive measurements and, in other words, that the illuminations should provide only the desorption of the captured $\mathrm{NO}_{2}$ without the undesirable drift of baseline or/ and NRM values. Regarding the influence of the temperature, it seems that the photoinitiation at higher temperatures resulted in cleaner films with lower concentration of adsorbates, thus becoming more attractive for $\mathrm{NO}_{2}$ capture. A higher amount of captured $\mathrm{NO}_{2}$ then needed a longer illumination period to get desorbed. Typical examples of raw 


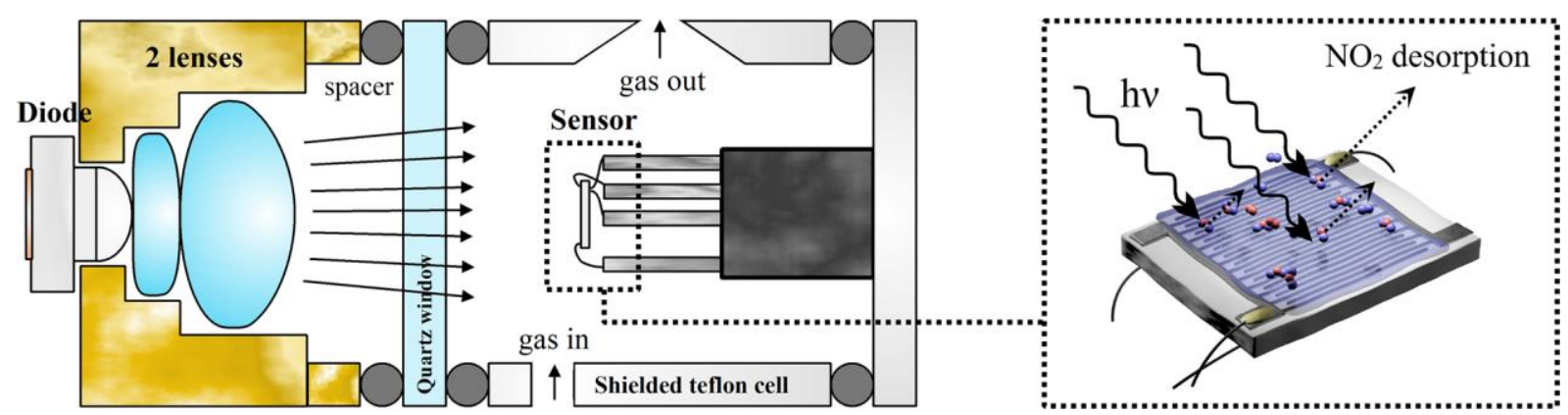

Figure 2. Illustration of the illumination setup. Sensors were illuminated by power LED diodes $(375-850 \mathrm{~nm})$ refocused by two lens optics to reach maximal spatial homogeneity of light intensity.
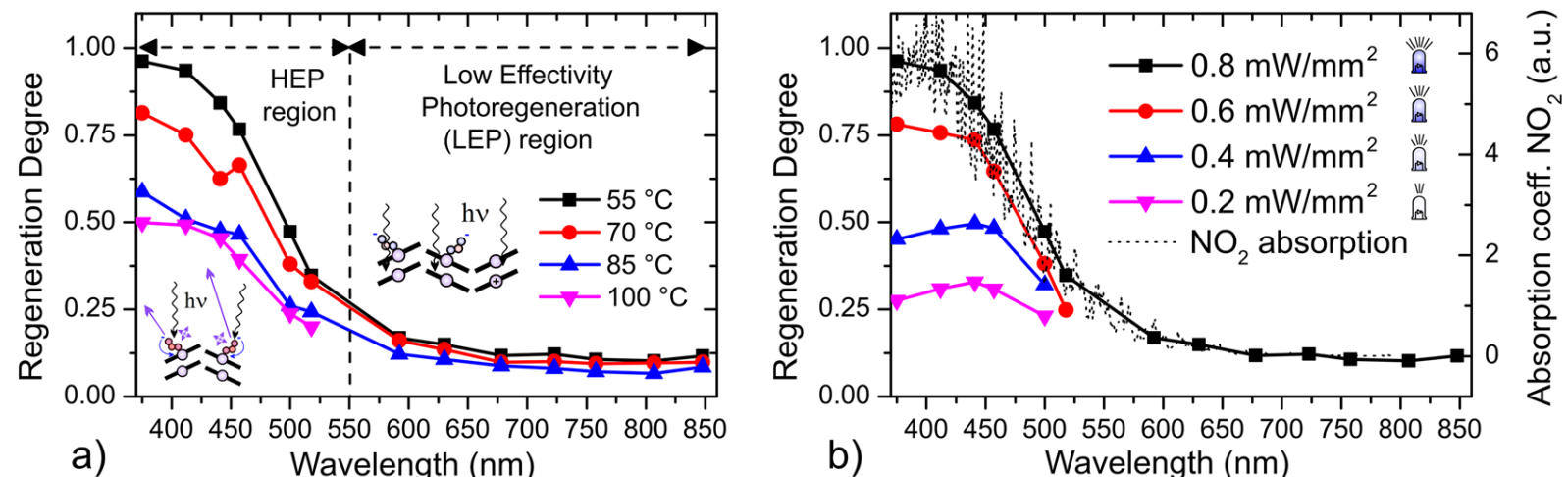

Figure 3. Dependence of the regeneration degree RD on the wavelength of the incident illumination and the influence of (a) sensor operating

temperature $\left(55,70,85\right.$, and $\left.100{ }^{\circ} \mathrm{C}\right)$ and $(\mathrm{b})$ intensity of illumination $(0.2,0.4,0.6$, and $0.8 \mathrm{~mW} / \mathrm{mm} 2)$. The absorption spectrum of $\mathrm{NO}_{2}$ in the

gaseous phase at $20^{\circ} \mathrm{C}$ (dotted line) was taken from the database (ref 33).

data are available in the Supporting Information (Figures S2 and S3).

Photoregeneration at Various Illumination Intensities. To

further investigate the nature of the photoregeneration processes, we carried out a series of measurements at a constant temperature of $55^{\circ} \mathrm{C}$ and various intensities of illumination $\left(0.2,0.4,0.6\right.$, and $\left.0.8 \mathrm{~mW} / \mathrm{mm}_{2}\right)$. During all these measurements, we also kept the constant baseline value of 310 $\pm 18 \mathrm{M} \Omega$. Under these circumstances, the RD was found to be more or less linearly proportional to the intensity of the illumination (Figure $3 \mathrm{~b}$ ). This finding is particularly promising for the applicability of the chemiresistors in practice as it should allow to easily adapt the photoregenerating effect to reproducibly restore the original sensor state after the exposures to various concentrations, ideally in one short photoregenerating step. The NRM was approximately constant $0.26 \pm 0.02$ for all sequences with no significant trend. 
Mechanism of Photoregeneration. Considering the

mechanism, two main types have been proposed for photoinduced molecular desorptions: (1) DIET (desorption or

dissociation induced by electronic transitions) and (2) LITD

(laser-induced thermal desorptions).34 In our case, the experimental evidence seems to disqualify the latter mechanism. The sensors were tempered at a selected temperature before exposure to light. At the beginning of the exposure (step 4 of the testing sequences described in the Experimental Section), the sensor heating power was fixed and the light was allowed to further heat the phthalocyanine layer by absorption. Differences in the layer temperature were proportional to the phthalocyanine absorption coefficient and are plotted in Figure 6. For $\mathrm{ZnPc}$, the maximal layer temperatures during the $" 55^{\circ} \mathrm{C}$ measurement" were $62{ }^{\circ} \mathrm{C}\left(55+7^{\circ} \mathrm{C}\right)$ for illumination by 0.8 $\mathrm{mW} / \mathrm{mm} 2$ of 723 or $592 \mathrm{~nm}$ due to the absorption in ZnPc's Q-band.15 Nevertheless, the regeneration degree was considerably lower for those wavelengths than for $441 \mathrm{~nm}$, for example, where the absorption of $\mathrm{ZnPc}$ is negligible and hence the temperature rise is smaller $\left(58.7^{\circ} \mathrm{C}\right.$, i.e., $\left.55+3.7^{\circ} \mathrm{C}\right)$.

This research finds a partial parallel in the class of inorganic semiconductors where UV-vis light can be used to activate the sensors to operate at ambient temperature. 35 It has been reported that the application of light can also accelerate the regeneration of such sensors based on the following model (we will refer to it as RecDes, recombinative desorption model). At first (1), the light generates a hole in a semiconductor's valence band exciting the electron to the conduction band. Second (2), the hole reacts with negatively charged chemisorbed species to release them.36 Extending the mechanism to chemisorbed $\mathrm{NO}_{2}$, we obtain eq 3 .

$$
\begin{aligned}
& h u \rightarrow e+h-+(1) \\
& h+\mathrm{O}_{2} \text { (ads) } \rightarrow \mathrm{O}_{2} \text { (gas) }+-(2) \\
& h+\mathrm{NO}_{2} \text { (ads) } \rightarrow \mathrm{NO}_{2} \text { (gas) }+-(3)
\end{aligned}
$$

In the end, the photoexcited electron from eq 1 is trapped in another hole because holes are major charge carriers in phthalocyanines as they are p-type semiconductors.37 This model is consistent with the increase in the resistance of $\mathrm{ZnPc}$ after illumination, which was observed regardless of whether the sensor was exposed to $\mathrm{NO}_{2}$ before. This model also predicts a threshold for the effective wavelengths as it requires sufficient energy to promote the electron across the band gap. The band gap was reported to be approximately $2 \mathrm{eV}$ for ZnPc, which corresponds to the wavelength of $620 \mathrm{~nm} .38$ This value was found close to the border of the HEP region (see Figure 3a).

Nevertheless, the tight correlation between the $\operatorname{RD}(\lambda)$ and $\mathrm{ANO}_{2}(\lambda)$ also suggests another model based on the direct absorption of light by the chemisorbed $\mathrm{NO}_{2}$ (NLA model). It was reported that $90 \%$ of light energy absorbed by $\mathrm{NO}_{2}$ is supposed to be converted to vibrational motion that could stimulate the rate of molecular desorption.39 These effects have been already used for photoacoustic detection of $\mathrm{NO}_{2}$ by amplitude-modulated high-power light emitting diodes.39 Both models would expect the proportionality between the light intensity and rate of $\mathrm{NO}_{2}$ desorption.

Sensitivity vs Concentration. The proposed method was 
further investigated in order to determine its $\mathrm{NO}_{2}$ detection limit. It was found out that the NRM is a linear function of $\mathrm{NO}_{2}$ concentration in the range from 0.2 to $0.9 \mathrm{ppm}$ with an estimated detection limit of $20 \mathrm{ppb}$. The detection limit was calculated according to the methodology reported in ref 40.
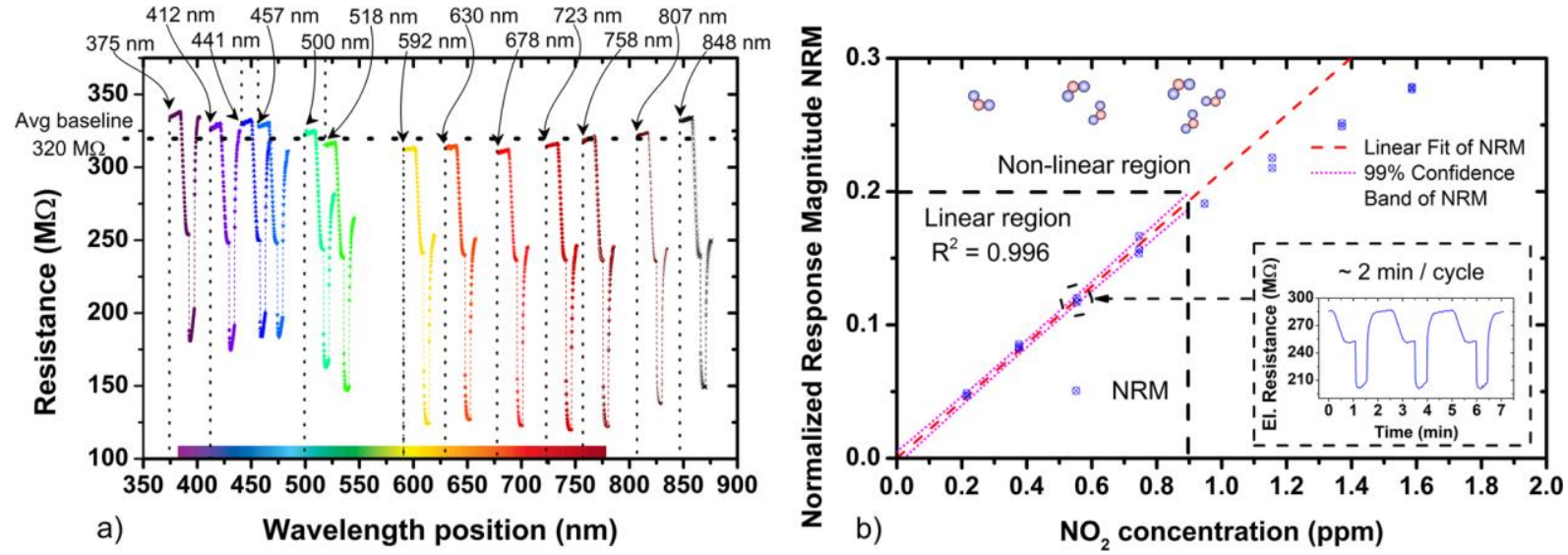

Figure 4. (a) Illustration of the stability of the baseline $320 \pm 9 \mathrm{M} \Omega$, constant value of the NRM $0.25 \pm 0.02$, and evolution of the regeneration

degree RD. The data consists of sequence steps 1-5 recorded during the measurement of $\mathrm{ZnPc}$ at $55^{\circ} \mathrm{C}$ illuminated by light of various

wavelengths $(0.8 \mathrm{~mW} / \mathrm{mm} 2)$. The wavelength position ( $\mathrm{x}$-axis) is an auxiliary variable proportional to time $(1 \mathrm{~nm}=6$ s). The beginning of each

sequence is positioned on the nominal wavelength of the LED used for the photoregeneration. (b) Dependence of the NRM on the concentration

of $\mathrm{NO}_{2}(0.2-1.6 \mathrm{ppm})$ for a $\mathrm{ZnPc}$ chemiresistor operating at $55^{\circ} \mathrm{C}$. The inset contains an example of the raw data recorded for the concentration

of $0.55 \mathrm{ppm}$ illustrating good repeatability and short measurement cycle duration. The photoregenerations were carried out by the $441 \mathrm{~nm}$ light.

This demonstrates the preservation of sensitivity achieved by the traditional "steady state approach" methods while the measurement cycle duration is significantly reduced ( $\sim 2$ vs

$\sim 15 \mathrm{~min}$ ).24 For the concentrations exceeding $0.9 \mathrm{ppm}$, the trend was still monotonic, but its slope was mildly decreasing (Figure 4b).

Impact of the Method on Sensor Power Consumption. To evaluate the impact of the proposed method on the sensor power demand, we first calculated the consumption of our chemiresistors operating according to the "standard" optimized protocol reported in ref 24 . Then we compared it with the consumption when following the protocol proposed in this article. The standard $15 \mathrm{~min}$ protocol for one measurement consists of 2 min of exposure to $\mathrm{NO}_{2}$ at $80^{\circ} \mathrm{C}, 12$ min of cleaning at $180^{\circ} \mathrm{C}$, and 1 min of stabilization again at $80^{\circ} \mathrm{C}$. For such a regime, the average power demand of our chemiresistors was calculated as $116 \mathrm{~mW}(3 / 15 \times 45 \mathrm{~mW}+$ $12 / 15 \times 134 \mathrm{~mW}$ ). According to the new method, the chemiresistors operated at $55^{\circ} \mathrm{C}$, consuming $23 \mathrm{~mW}$ for 14.5 $\mathrm{min}$ and $121 \mathrm{~mW}(23+98 \mathrm{~mW})$ for $0.5 \mathrm{~min}$ during the photoregeneration step with the $441 \mathrm{~nm}$ LED. As a result, the average consumption was ca. $26 \mathrm{~mW}$, which was more than 4 
times better when compared with the standard protocol. The consumption could be even better after improving the focus optics, as only ca. $4 \mathrm{~mW}\left(0.8 \mathrm{~mW} / \mathrm{mm}_{2} \times 5 \mathrm{~mm} 2\right)$ is needed to promote the photoregeneration.

Photoregeneration of the Other Phthalocyanine

Chemiresistors ( $\mathrm{CuPc}, \mathrm{H}_{2} \mathrm{Pc}, \mathrm{PbPc}$, and $\mathrm{FePc}$ ) at the

Temperature of $55^{\circ} \mathrm{C}$. Photoregeneration of CuPc

Chemiresistors. CuPc chemiresistors, with the baseline set at

$106 \pm 2 \mathrm{M} \Omega$, did not photoregenerate with wavelengths

greater than $550 \mathrm{~nm}(\mathrm{RD} \sim 0 \%)$. The photoregeneration was

observed first with $518 \mathrm{~nm}(\mathrm{RD}=37 \%)$ and with even shorter wavelengths it was more and more effective (RD $=58-244 \%$, Figure 5). The trend was analogous to the one observed for $\mathrm{ZnPc}$, but the difference of the photoregeneration effectivities between the LEP and HEP regions was greater. The highest achieved RD values indicate that, in some cases, it was possible to "overregenerate" the chemiresistor set at a certain baseline while fulfilling the stability criterion of $\Delta R / R \leq 1 \% / \mathrm{min}$. The "overregeneration" was probably caused by the photoinduced desorption of some part of the chemisorbed $\mathrm{O}_{2}$ or by the photoinduced desorption of the residual traces of $\mathrm{NO}_{2}$, which were not removed during the photoinitiation procedure described in the Experimental Section. However, the "overregeneration" is not a desirable effect, because it brings an issue connected with the requirement of the preservation of sensor baseline.

The baseline value should be conserved for all the measurements to ensure the preservation of a specific film state necessary for correct comparison of consecutive detections and regenerations. In this case, the "overregeneration" was compensated by inserting an extra cycle with the same exposition to $\mathrm{NO}_{2}$ but followed by the photoregeneration with a lower illumination intensity or shorter illumination time. This, with regard to the dependence of the RD on the illumination intensity discussed above and a similar dependence on time, allowed return to the original baseline value while preserving the sensor sensitivity, because the NRM was approximately constant $0.61 \pm 0.04$ for all sequences with no trend as in the case of ZnPc. To complete this point, it should be noted that the direct return to the original baseline after the "overregeneration" solely by the exposure to the $\mathrm{NO}_{2}$ gas (without the subsequent illumination) did not provide comparable results as the NRM was decreasing proportionally to the dose of $\mathrm{NO}_{2}$ used for the baseline compensation. Photoregeneration of PbPc Chemiresistors. PbPc chemiresistors, working at the baseline of $15.3 \pm 0.2 \mathrm{M} \Omega$, did not

significantly photoregenerate with any wavelength in the range from 375 to $850 \mathrm{~nm}(\mathrm{RD}=-3-17 \%$, Figure 5$)$. As the photoregeneration was not effective in this case, we had to use the classical thermal recovery mechanism to regenerate the sensors. After 10 steps of illumination for each wavelength, step 4 in our methodology was replaced by a temporal increase of the operating temperature. It was increased from 55 to 130 ${ }^{\circ} \mathrm{C}$ for $30 \mathrm{~s}$, and it went back to $55^{\circ} \mathrm{C}$ to check the progress of the regeneration. With this approach, we managed to get an approximately constant NRM of $0.68 \pm 0.02$ for all sequences 
with no trend as in the case of other phthalocyanines.

However, we encountered increasing instability of sensor baselines. This instability had to be compensated, so it resulted in dramatic prolongations of settling times. Nevertheless, despite these prolongations, it was problematic to fulfill the stability criterion defined in the experimental section. Due to these effects, PbPc cannot be considered as a suitable material for gas detection by our method.

Photoregeneration of FePc Chemiresistors. As opposed to $\mathrm{PbPc}, \mathrm{FePc}$ chemiresistors, operating at the baseline of $1.050 \pm$ $0.002 \mathrm{G} \Omega$, photoregenerated with all the wavelengths $(\mathrm{RD} \gg$ $0 \%$ ) and the RD significantly increased with descending wavelength as for $\mathrm{ZnPc}$ and $\mathrm{CuPc}$ (see Figure 5). However, the regeneration was much more intensive. For the wavelengths $678 \mathrm{~nm}+$, the RD varied in the range from $30 \%$ to $99 \%$. Then it rose to $156 \%$ for $630 \mathrm{~nm}$ and to several thousand percent for the light of 518-375 $\mathrm{nm}$. These huge "overregenerations" complicated the measurement as the sensors were returning to their baselines very slowly (one return took practically several hours or days). To compensate this, we used controlled $\mathrm{NO}_{2}$ expositions to speed up the baseline return (without the subsequent illumination). Probably as a result of this approach, the NRM was mildly decreasing in the sequence of the experiments. At the end, it had $50 \%$ of its original magnitude. In spite of this, the application of $\mathrm{FePc}$ for $\mathrm{NO}_{2}$ detection seems to be prospective in combination with either longer wavelengths or significantly lower intensities $(\sim 0.01 \mathrm{~mW} /$ $\mathrm{mm} 2$ ) of the shorter wavelengths.

Photoregeneration of $\mathrm{H}_{2} \mathrm{Pc}$ Chemiresistors. $\mathrm{H}_{2} \mathrm{Pc}$ chemiresistors, with the baseline set at $176 \pm 26 \mathrm{G} \Omega$, did not

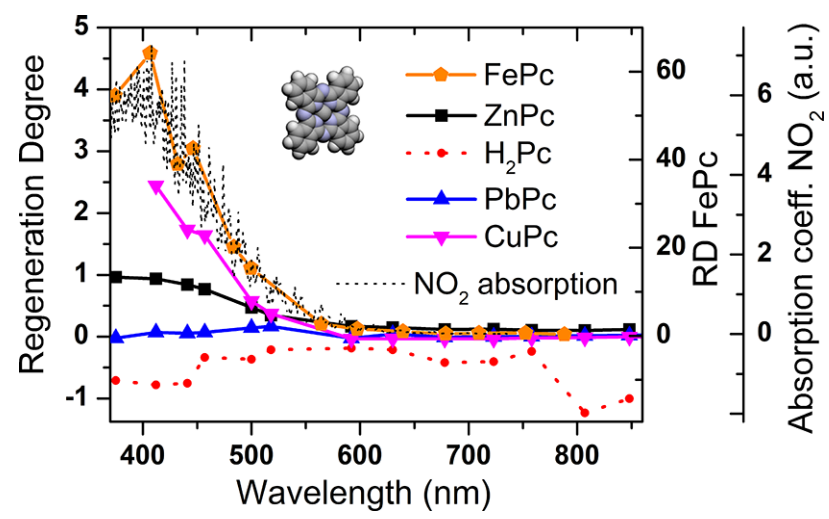

Figure 5. Dependence of the regeneration degree RD on the phthalocyanine material (FePc, $\mathrm{ZnPc}, \mathrm{H}_{2} \mathrm{Pc}, \mathrm{PbPc}$, and $\mathrm{CuPc}$ ). The absorption spectrum of $\mathrm{NO}_{2}$ in the gaseous phase at $20{ }^{\circ} \mathrm{C}$ (dotted line) was taken from the database (ref 33).

satisfactorily photoregenerate with any wavelength in the range measured. In terms of the RD, the values obtained were even negative in the range from $-124 \%$ to $-18 \%$. The reason for the negative values could be that illumination causes a temporal increase in the layer conductivity and generates new free charge carriers, which seem to have much longer lifetime in $\mathrm{H}_{2} \mathrm{Pc}$ than in the other phthalocyanines. This could 
be connected with $\mathrm{H}_{2} \mathrm{Pc}^{\prime}$ s significantly higher resistance baseline. So, at the time of the RD evaluation, some of these photocarriers were probably still present there (see Figure S4). A similar effect has already been observed for amorphous VOPC and named as persistent photoconductivity (PPC).41 If we waited a longer time before the evaluation of the photoregeneration (30 min, not $30 \mathrm{~s}$ as defined in the methodology), we obtained small positive values of the RD, mostly close to $0 \%$ (Figure S4). The inefficiency of the photoregeneration in the case of $\mathrm{H}_{2} \mathrm{Pc}$ forced us to apply thermal regeneration by temporal increases of the operating temperature to $90{ }^{\circ} \mathrm{C}$. The NRM was approximately constant, equal to $0.28 \pm 0.04$ with no statistically significant trend. Comparison, Implications for the Mechanism. Extending the study to the phthalocyanine materials other than $\mathrm{ZnPc}$ revealed the importance of the central cation for the process of photoregeneration. The phthalocyanines ( $\mathrm{ZnPc}, \mathrm{CuPc}, \mathrm{H}_{2} \mathrm{Pc}$, $\mathrm{PbPc}$, and $\mathrm{FePc}$ ) divided into two groups differing in the ability to photoregenerate. $\mathrm{ZnPc}$, CuPc, and FePc exhibited photoregeneration; $\mathrm{H}_{2} \mathrm{Pc}$ and $\mathrm{PbPc}$ did not. This division seems to correspond with the symmetry of the molecules. $\mathrm{Zn}_{2+}, \mathrm{Cu}_{2+}$, and $\mathrm{Fe}_{2+}$ cations are small and fit the cavity of the macrocycle, letting the phthalocyanine preserve its planar structure with the symmetry $\mathrm{D}_{4}$. $\mathrm{H}_{2} \mathrm{Pc}^{\prime}$ s hydrogens reduce its symmetry to $\mathrm{D}_{2} \mathrm{~h}$ and $\mathrm{PbPc}^{\prime}$ s large $\mathrm{Pb}_{2+}$ cation that distorts the planarity of the molecule reduces the symmetry to $\mathrm{C}_{4 \mathrm{v} .15}$ Regarding the mechanism of desorption, from the viewpoint of the models considered above, the universal shape of the $\operatorname{RD}(\lambda)$ for various phthalocyanines (it differs just by a multiplicative scaling factor) seems to favor the one based on the direct absorption of light by the chemisorbed $\mathrm{NO}_{2}$. The RecDes model would expect some shifts of the $\operatorname{RD}(\lambda)$ curve based on the variations of the band gaps (CuPc $~ 1.7 \mathrm{eV}) .42$ However, the precise value of the band gap widths may be difficult to consider as the literature contains rather a range of values for each phthalocyanine.

Stability of the Phthalocyanine Sensitive Layers

under Illumination (Spectral Study). UV-vis spectra and FTIR spectra of the layers were consistent with those in the literature. The comparison is provided in the Supporting Information.

Effects of the Illuminations on UV-Vis Spectra of the Phthalocyanine Layers. The Pc spectra did not change significantly with the layer illuminations at working conditions for photoregeneration ( $441 \mathrm{~nm}, 0.8 \mathrm{~mW} / \mathrm{mm} 2,2 \mathrm{~h}$ ). Based on these findings, we are convinced that, under the working conditions, there are no irreversible changes in the layers caused by the illuminations. We believe that the effects of light are restricted only to (i) the photostimulated desorption of the species adsorbed on the surface of the phthalocyanine layers $\left(\mathrm{NO}_{2}, \mathrm{O}_{2}, \mathrm{H}_{2} \mathrm{O}\right)$ and (ii) the generation of photoinduced mobile charge carriers. However, we observed some changes of FePc and PbPc UV-vis spectra after stronger illuminations $\left(7.3 \mathrm{~mW} / \mathrm{mm}_{2}, 1 \mathrm{~h}\right)$. In these particular cases, the stronger illuminations led to small depreciations of the $\mathrm{Q}$ absorption bands (626 nm, $-6 \% ; 719 \mathrm{~nm},-3 \%$, respectively, Figure 6). We think that these depreciations were caused by partial temperature-stimulated recrystallizations of the FePc and PbPc 
layers, as those layers exhibit some level of absorption of the $441 \mathrm{~nm}$ light. The temperature rose by more than several tens of degrees $\left({ }^{\circ} \mathrm{C}\right)$ for the stronger illuminations and hence could provide the energy to the layers to recrystallize. $\mathrm{PbPc}$, for example, is known to have two polymorphs differing in their absorption spectra: (i) monoclinic with the absorption maxima at $740 \mathrm{~nm}$ and (ii) triclinic with the maxima at $900 \mathrm{~nm} .43$ It has been reported that the annealing process can induce the recrystallization of the as-deposited monoclinic phases to the more thermodynamically stable triclinic form.43 On the contrary, the absorption of the layers of the other phthalocyanines ( $\mathrm{ZnPc}, \mathrm{CuPc}$, and $\mathrm{H}_{2} \mathrm{Pc}$ ) is negligible in the spectral region around $441 \mathrm{~nm}$ and thus their temperature rises were insufficient to alter layer morphology.

Effects of the Illuminations on FTIR Spectra of the Phthalocyanine Layers. The FTIR spectra remained unchanged for the working intensity of the illuminations $(0.8$ $\mathrm{mW} / \mathrm{mm}_{2}$ ), supporting the interpretation mentioned above and the claim of stability. After the stronger illuminations (7.3 $\mathrm{mW} / \mathrm{mm}_{2}$ ), we again recorded the almost identical spectra containing only minor changes of very weak bands for $\mathrm{ZnPc}$ at 668 or $434 \mathrm{~cm}-1$ and CuPc at $571 \mathrm{~cm}-1$ (Figure S5). A possible explanation of these changes may lie in the stimulated desorption of several adsorbates $\left(\mathrm{NO}_{2}, \mathrm{O}_{2}, \mathrm{H}_{2} \mathrm{O}\right)$ which are present in phthalocyanine layers at ambient conditions.

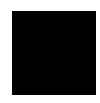

\section{CONCLUSIONS}

ZnPc, CuPc, and FePc thin layers containing certain amounts of $\mathrm{NO}_{2}$ as a result of a chemiresistive detection process were found to photoregenerate when they were exposed to light with a wavelength shorter than $550 \mathrm{~nm}$. Such light demonstrated its capability to stimulate $\mathrm{NO}_{2}$ desorption from the layer, accelerating its recovery to the original state

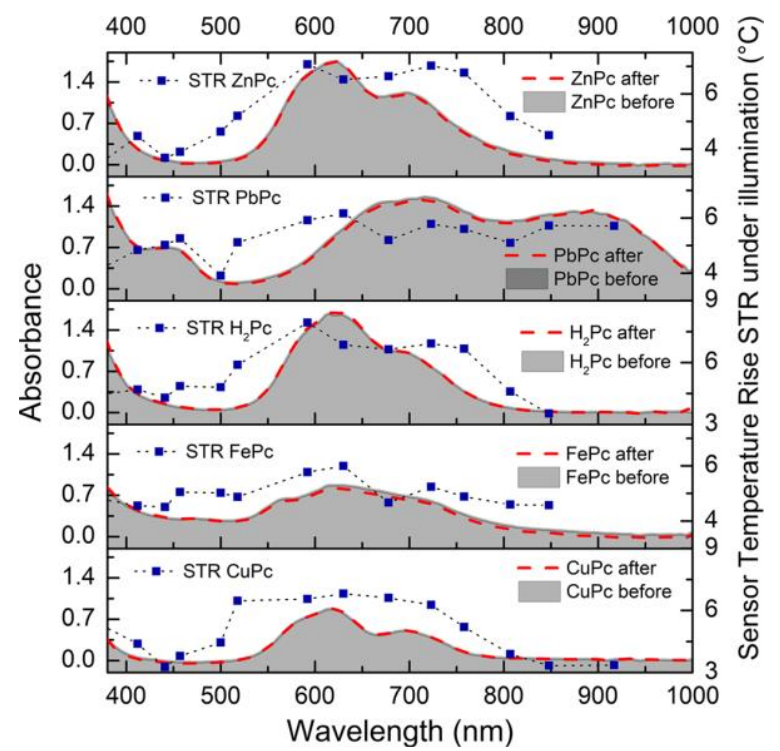

Figure 6. UV-vis spectra of the phthalocyanine sensitive layers before (gray area) and after (red dashed curve) illumination $(7.3 \mathrm{~mW} / \mathrm{mm} 2$, $1 \mathrm{~h}$ ). The graphs contain superimposed curves (STR) illustrating temperature effects of illumination on the chemiresistors $(0.8 \mathrm{~mW} /$ $\mathrm{mm} 2$ ). 
before the detection of $\mathrm{NO}_{2}$. This effect was ascribed either to the generation of holes in the organic semiconductor that can recombine with the chemisorbed species or to the direct desorption induced by electronic transitions originating from the light absorption in chemisorbed $\mathrm{NO}_{2}$. It was shown that photoregeneration can be conducted at ambient or slightly elevated temperatures $\left(55-100^{\circ} \mathrm{C}\right)$, thus opening the possibility of simple phthalocyanine based chemiresistors operating at low temperatures. Lower temperatures imply lower electrical consumption (ca. 25\%) and longer lifetime of the sensors.

Moreover, we modified the kinetic operating procedure suggested in ref 25 to benefit not only from the considerable acceleration of measurement (T meas.cycle was $\sim 2 \mathrm{~min}$, more than 7 times faster than the traditional optimized approach presented in ref 24), but also from the preservation of the limit of detection $(<20 \mathrm{ppb})$ and the improved baseline stability $(\rightarrow$ better repeatability of the measurement). The photoregeneration effects were further found to be approximately linearly dependent on the light intensity and also proportional to the operating temperature and the phthalocyanine type.

From a practical standpoint, it has been shown that photoregeneration could help phthalocyanine based sensors to become more than a viable alternative to conventional techniques for $\mathrm{NO}_{2}$ monitoring.

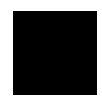

\section{ASSOCIATED CONTENT}

\section{* Supporting Information}

The Supporting Information is available free of charge on the ACS Publications website at DOI: 10.1021/acssensors.

8b00922.

Evaporation parameters and details about the sensitive layers; details about the illumination; typical raw data of ZnPc photoregeneration; persistent photoconductivity of $\mathrm{H}_{2} \mathrm{Pc}$; UV-vis and FTIR spectra of the sensitive layers and their comparison with the literature; effects of the illuminations on FTIR spectra of the phthalocyanine layers (PDF)

Animated video of the photoregeneration (AVI)

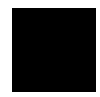

\section{AUTHOR INFORMATION}

\section{Corresponding Author}

*E-mail: vrnatam@vscht.cz.

ORCID

Martin Vrnata: 0000-0002-1199-1458

Notes

The authors declare no competing financial interest. 


\section{ACKNOWLEDGMENTS}

This work was supported by Czech Science Foundation (GACR), Projects No. 17-13427S and No. 18-09347S and also by Ministry of Education, Youth and Sports within the projects LTC17058 and 8J18FR011 and by COST Action CA15107 MultiComp. Financial support from specific university research (MSMT No 21-SVV/2018) and PHC Barrande 2018 (40672RL) is also acknowledged.

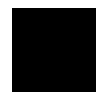

REFERENCES

(1) Kim, I.-D.; Rothschild, A.; Lee, B. H.; Kim, D. Y.; Jo, S. M.; Tuller, H. L. Ultrasensitive Chemiresistors Based on Electrospun $\mathrm{TiO}_{2}$ Nanofibers. Nano Lett. 2006, 6 (9), 2009-2013.

(2) Zhang, D.; Liu, Z.; Li, C.; Tang, T.; Liu, X.; Han, S.; Lei, B.; Zhou, C. Detection of $\mathrm{NO}_{2}$ down to ppb Levels Using Individual and Multiple In2 $\mathrm{O}_{3}$ Nanowire Devices. Nano Lett. 2004, 4 (10), 19191924.

(3) Campbell, M. G.; Liu, S. F.; Swager, T. M.; Dinca, M. Chemiresistive Sensor Arrays from Conductive 2D Metal-Organic Frameworks. J. Am. Chem. Soc. 2015, 137 (43), 13780-13783.

(4) Assen, A. H.; Yassine, O.; Shekhah, O.; Eddaoudi, M.; Salama, K. N. MOFs for the Sensitive Detection of Ammonia: Deployment of fcu-MOF Thin Films as Effective Chemical Capacitive Sensors. ACS Sensors 2017, 2 (9), 1294-1301.

(5) Yuan, W.; Huang, L.; Zhou, Q.; Shi, G. Ultrasensitive and Selective Nitrogen Dioxide Sensor Based on Self-Assembled Graphene/Polymer Composite Nanofibers. ACS Appl. Mater. Interfaces 2014, 6 (19), 17003-17008.

(6) Cho, S.-Y.; Cho, K. M.; Chong, S.; Park, K.; Kim, S.; Kang, H.; Kim, S. J.; Kwak, G.; Kim, J.; Jung, H.-T. Rational Design of Aminopolymer for Selective Discrimination of Acidic Air Pollutants. ACS Sensors 2018, 3 (7), 1329-1337.

(7) Pavluchenko, A. S.; Mamykin, A. V.; Kukla, A. L.; Konoshchuk, N. V.; Posudievsky, O. Y.; Koshechko, V. G. Estimation of multicomponent organic solvent vapor mixture composition with electroconducting polymer chemiresistors. Sens. Actuators, B 2016 , 232, 203-218.

(8) Evyapan, M.; Dunbar, A. D. F. Improving the selectivity of a free base tetraphenylporphyrin based gas sensor for $\mathrm{NO}_{2}$ and carboxylic acid vapors. Sens. Actuators, B 2015, 206, 74-83.

(9) Surwade, S. P.; Agnihotra, S. R.; Dua, V.; Manohar, S. K. Nitrogen dioxide vapor detection using poly-o-toluidine. Sens. Actuators, B 2009, 143 (1), 454-457.

(10) Othman, M.; The 'ron, C.; Bendahan, M.; Caillat, L.; Rivron, C.; Bernardini, S.; Le Chevallier, G.; Chevallier, E.; Som, M. P.; Aguir, K.; Tran-Thi, T. H. Efficiency of new ozone filters for $\mathrm{NO}_{2}$ sensing and air depollution. Sens. Actuators, B 2018, 265, 591-599.

(11) Fitl, P.; Vrnata, M.; Kopecky, D.; Vlcek, J.; Skodova, J.; Bulir, J.; Novotny, M.; Pokorny, P. Laser deposition of sulfonated phthalocyanines for gas sensors. Appl. Surf. Sci. 2014, 302, 37-41. (12) Lee, Y.-L.; Sheu, C.-Y.; Hsiao, R.-H. Gas sensing characteristics of copper phthalocyanine films: effects of film thickness and sensing temperature. Sens. Actuators, B 2004, 99 (2), 281-287.

(13) Tomec `ek, D.; Fitl, P.; Mares`ova', E.; Vlc ek, J.; Hofmann, J.; Vrn ata, M. Silver phthalocyanine thin films carrying gold, palladium 
and silver nanoparticles for detection of taggants in explosives. Thin Solid Films 2017, 630, 31-37.

(14) Zhou, R.; Josse, F.; Go pel, W.; O“"ztu“ rk, Z. Z.; Bekarog`lu, O”. Phthalocyanines as sensitive materials for chemical sensors. Appl. Organomet. Chem. 1996, 10 (8), 557-577.

(15) Kadish, K. M.; Smith, K. M.; Guilard, R. The Porphyrin Handbook: Phthalocyanines: properties and materials; Academic Press: 2000.

(16) Guillaud, G.; Simon, J.; Germain, J. P. Metallophthalocyanines Gas sensors, resistors and field effect transistors. Coord. Chem. Rev. 1998, 178-180, 1433-1484.

(17) Park, J. H.; Royer, J. E.; Chagarov, E.; Kaufman-Osborn, T.; Edmonds, M.; Kent, T.; Lee, S.; Trogler, W. C.; Kummel, A. C.

Atomic imaging of the irreversible sensing mechanism of $\mathrm{NO}_{2}$ adsorption on copper phthalocyanine. J. Am. Chem. Soc. 2013, 135 (39), 14600-14609.

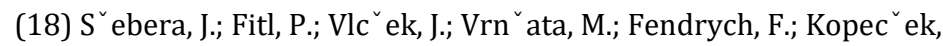
J.; Kratochvílova', I. Interaction of selected gases with zinc phthalocyanine thin films: Theoretical and experimental studies. Eur. Phys. J.: Appl. Phys. 2013, 64 (1), 10202.

(19) Słota, R.; Dyrda, G.; Hnatejko, Z.; Karolczak, J.; Stryła, Z. Effect of air-absorbed oxygen and moisture on the chemical stability of photoexcited $\mathrm{Mg}$, Zn and Eu phthalocyanines in dimethylformamide. J. Porphyrins Phthalocyanines 2006, 10 (1), 43-54.

(20) Gadenne, V.; Porte, L.; Patrone, L. Structure and growth mechanism of self-assembled monolayers of metal protoporphyrins and octacarboxylphthalocyanine on silicon dioxide. RSC Adv. 2014, 4 (110), 64506-64513.

(21) Novotny' , M.; S` ebera, J.; Bensalah-Ledoux, A.; Guy, S.; Bulír ` , J.;

Fitl, P.; Vlc ěek, J.; Za 'kutna', D.; Mareš̌ova', E.; Hubík, P.; Kratochvílova',

I.; Vrn `ata, M.; Lanc ${ }^{2}$ ok, J. The growth of zinc phthalocyanine thin films by pulsed laser deposition. J. Mater. Res. 2016, 31 (1), 163-172.

(22) Pizzini, S.; Timo, G. L.; Beghi, M.; Butta, N.; Mari, C. M.;

Faltenmaier, J. Influence of the structure and morphology on the sensitivity to nitrogen oxides of phthalocyanine thin-film resistivity sensors. Sens. Actuators 1989, 17, 481-491.

(23) Lee, Y. L.; Sheu, C. Y.; Hsiao, R. H. Gas sensing characteristics of copper phthalocyanine films: Effects of film thickness and sensing temperature. Sens. Actuators, B 2004, 99 (2-3), 281-287.

(24) Brunet, J.; Garcia, V. P.; Pauly, A.; Varenne, C.; Lauron, B. An optimized gas sensor microsystem for accurate and real-time measurement of nitrogen dioxide at ppb level. Sens. Actuators, B 2008, 134 (2), 632-639.

(25) Wilson, A.; Wright, J. D.; Chadwick, A. V. A microprocessorcontrolled nitrogen dioxide sensing system. Sens. Actuators, B 1991, 4

(3), 499-504.

(26) Zhou, Q.; Gould, R. D. A study of the response rate to nitrogen dioxide exposure in metal phthalocyanine thin film sensors. Thin Solid Films 1998, 317 (1), 436-439.

(27) Jalil, A. R.; Chang, H.; Bandari, V. K.; Robaschik, P.; Zhang, J.; Siles, P. F.; Li, G.; Burger, D.; Grimm, D.; Liu, X.; Salvan, G.; Zahn, D. R. T.; Zhu, F.; Wang, H.; Yan, D.; Schmidt, O. G. Fully Integrated Organic Nanocrystal Diode as High Performance Room Temperature NO2 Sensor. Adv. Mater. 2016, 28 (15), 2971-2977.

(28) Newton, M. I.; Starke, T. K. H.; Willis, M. R.; McHale, G. NO2 detection at room temperature with copper phthalocyanine thin film devices. Sens. Actuators, B 2000, 67 (3), 307-311.

(29) Sadaoka, Y.; Jones, T. A.; Go“ pel, W. Fast $\mathrm{NO}_{2}$ detection at room temperature with optimized lead phthalocyanine thin-film structures. Sens. Actuators, B 1990, 1 (1), 148-153.

(30) Baldini, F.; Capobianchi, A.; Falai, A.; Mencaglia, A. A.; 
Pennesi, G. Reversible and selective detection of $\mathrm{NO}_{2}$ by means of optical fibres. Sens. Actuators, B 2001, 74 (1), 12-17.

(31) Bueno, A.; Lahem, D.; Caucheteur, C.; Debliquy, M. Reversible $\mathrm{NO}_{2}$ Optical Fiber Chemical Sensor Based on LuPc2 Using Simultaneous Transmission of UV and Visible Light. Sensors 2015, 15 (5), 9870.

(32) Tomec `ek, D.; Fitl, P.; Vlc `ek, J.; Mares`ova’ , E.; Vrn``ata, M. Detection of taggants in explosives on nanostructured metal/silver phthalocyanine chemiresistors: Influence of analyte photoactivation. Sens. Actuators, B 2017, 239, 147-156.

(33) Keller-Rudek, H.; Moortgat, G. K.; Sander, R.; So“rensen, R. The MPI-Mainz UV/VIS Spectral Atlas of Gaseous Molecules of Atmospheric Interest. Earth Syst. Sci. Data 2013, 5 (2), 365-373. (34) Kawasaki, T.; Mochida, T.; Katada, J. I.; Okahata, Y. Laser response of a quartz crystal microbalance: Frequency changes induced by light irradiation in the air phase. Anal. Sci. 2009, 25 (9), 10691075.

(35) Park, S.; An, S.; Mun, Y.; Lee, C. UV-enhanced $\mathrm{NO}_{2}$ gas sensing properties of $\mathrm{SnO}_{2}$-Core/ZnO-shell nanowires at room temperature. ACS Appl. Mater. Interfaces 2013, 5 (10), 4285-4292.

(36) Zhang, C.; Boudiba, A.; De Marco, P.; Snyders, R.; Olivier, M.G.; Debliquy, M. Room temperature responses of visible-light illuminated $\mathrm{WO}_{3}$ sensors to $\mathrm{NO}_{2}$ in sub-ppm range. Sens. Actuators, B 2013, 181, 395-401.

(37) Bassoul, P.; Toupance, T.; Simon, J. Semiconductivity and gassensing properties of crown-ether-substituted lutetium bisphthalocyanines. Sens. Actuators, B 1995, 26 (1), 150-152.

(38) Senthilarasu, S.; Velumani, S.; Sathyamoorthy, R.; Subbarayan, A.; Ascencio, J. A.; Canizal, G.; Sebastian, P. J.; Chavez, J. A.; Perez, R. Characterization of zinc phthalocyanine ( $\mathrm{ZnPc}$ ) for photovoltaic applications. Appl. Phys. A: Mater. Sci. Process. 2003, 77 (3), 383-389.

(39) Saarela, J.; Sorvaja“ rvi, T.; Laurila, T.; Toivonen, J. Phasesensitive method for background-compensated photoacoustic detection of $\mathrm{NO}_{2}$ using high-power LEDs. Opt. Express 2011, 19 (S4),

A725-A732.

(40) Loock, H.-P.; Wentzell, P. D. Detection limits of chemical sensors: Applications and misapplications. Sens. Actuators, B 2012, 173, 157-163.

(41) Santos, L. F.; Faria, R. M.; Del Can o, T.; de Saja, J. A.;

Constantino, C. J. L.; Amorim, C. A.; Mergulha ̃o, S. Observation of persistent photoconductivity in vanadyl phthalocyanine. J. Phys. D: Appl. Phys. 2008, 41 (12), 125107.

(42) So, F. Organic Electronics: Materials, Processing, Devices and Applications; CRC Press: 2009.

(43) Vasseur, K.; Rand, B. P.; Cheyns, D.; Froyen, L.; Heremans, P. Structural evolution of evaporated lead phthalocyanine thin films for near-infrared sensitive solar cells. Chem. Mater. 2011, 23 (3), 886895. 